\title{
Case 1 - Stage IV low-grade serous ovarian carcinoma management in an elderly patient
}

\author{
Luisa Sánchez-Lorenzo ${ }^{1}$, Antonio González-Martín ${ }^{2}$ \\ Commentary: David M. Gershenson
}

\begin{abstract}
Recurrent low-grade serous ovarian cancer (LGSOC) is considered relatively chemoresistant; until more effective chemotherapy approaches become available, surgical management remains the mainstay of treatment. However, some subtypes of LGSOC behave distinctly differently from high-grade SOC. The upregulation of estrogen and progesterone receptors in ovarian cancer suggests hormone therapy as a possible treatment modality. Our patient was a 75-year-old woman with advanced low-grade papillary serous ovarian cancer. In consultation, supported by appropriate histopathological examination and immunohistochemical study to guide the treatment decisions, our Gynaecological Oncology Multi-Disciplinary Team recommended primary debulking surgery. The patient underwent surgery followed by hormone therapy with letrozole, after declining bevacizumab-containing adjuvant chemotherapy. Eighteen months of ongoing monitoring has shown no signs of progressive disease and stable tumor markers. This case highlights the importance of initial assessment by a multidisciplinary team experienced in gynecological oncology, together with knowledge of the tumor biology, to determine effective treatment strategies for the management of advanced LGSOC.
\end{abstract}

Key words: low-grade serous ovarian cancer, surgery, chemotherapy, hormone therapy

\section{Introduction}

Low-grade serous ovarian cancer (LGSOC) accounts for between $5-10 \%$ of all serous ovarian carcinomas [1]. A decade of using the binary grading system for serous carcinoma [2] had to pass until in 2014 the World Health Organization changed the classification system of ovarian cancer in order to differentiate LGSOC and high-grade serous ovarian cancer (HGSOC) as two distinct entities [3]. Clinically, LGSOC is characterized by younger age, indolent clinical course and poor response to conventional chemotherapy, but is not refractory to platinum-based chemotherapy in any setting [1, 4-5]. Immunohistochemically it shows a low Ki-67 proliferation index and high levels of estrogen receptors (ER) compared with HGSOC. TP53 wild-type pattern can be useful to distinguish LGSOC from HGSOC. Genetic profiling has demonstrated frequent BRAF, NRAS, and KRAS mutations, in approximately $50-60 \%$ of the cases $[6,7]$.

This case emphasizes the importance of the knowledge of the tumor biology in order to be able to offer our patients different treatment strategies.

\section{Case description}

A 75-year-old woman underwent her annual gynecological review, where transvaginal sonography showed bilateral adnexal mixed solid and cystic masses of $6 \times 5 \mathrm{~cm}$ and $7 \times 5 \mathrm{~cm}$. The patient's medical history included fa- milial hypercholesterolemia and appendectomy in her twenties. Her family history included a sister diagnosed with breast cancer at 60 years of age. Laboratory tests reported a carcinoembryonic antigen (CA)-125 level of of $9407 \mathrm{IU} / \mathrm{mL}$ (normal value $<32 \mathrm{IU} / \mathrm{mL}$ ) and CA-15-3 level of $87.5 \mathrm{IU} / \mathrm{mL}$ (normal value $<35 \mathrm{IU} / \mathrm{mL}$ ).

A positron emission tomography/computed tomography (PET/CT) scan showed a high fluorodeoxyglucose (FDG) avidity pelvic mass of $10 \times 10 \mathrm{~cm}$ with multiple abdominal-pelvic implants, inguinal, retroperitoneal, bilateral internal mammary and left supraclavicular lymph nodes (Figures 1 and 2).

The case was discussed in the Gynaecological Oncology Multi-Disciplinary Team (MDT) meeting. As the supradiaphragmatic tumor load was low, we considered per-

${ }^{1}$ Medical Oncology Department and Gynecologic Oncology Unit, MD Anderson Cancer Center Hospital, Madrid, Spain.

${ }^{2}$ Clinica Universidad de Navarra, Madrid, Spain.

Correspondence to:

Antonio González Martin, MD,

Clinica Universidad de Navarra,

C/General Lopez Pozas 10, 28036 Madrid, Spain.

Phone: +34 913531920

E-mail: agonzalezm@seom.org

CANCER BREAKING NEWS 2017;5(2):39-45

DOI: $10.19156 / C B N .2017 .0047$ 

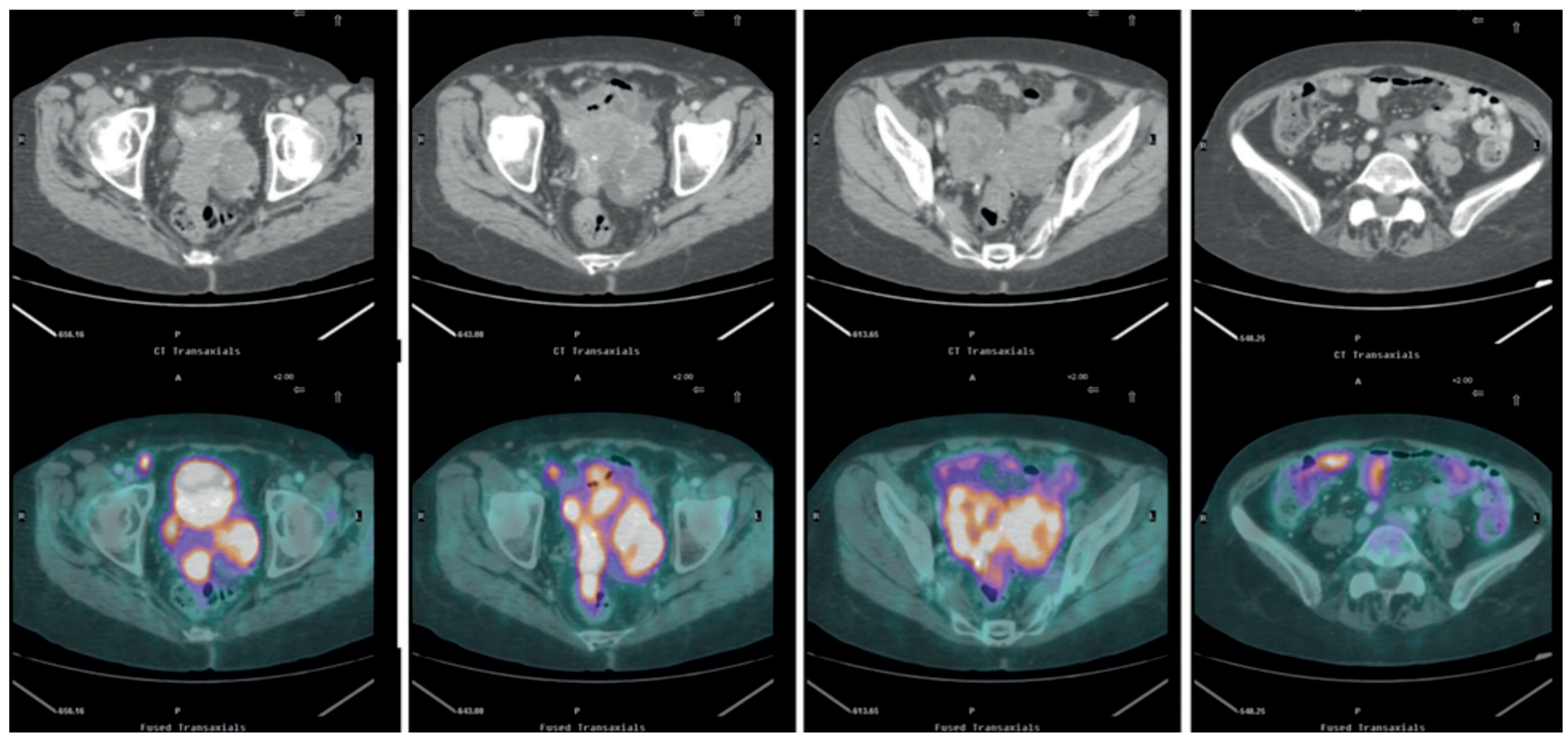

Fig. 1. Baseline positron emission tomography/computed tomography (PET/CT) scan: polylobulated heterogeneous mass within the pelvis of $10 \times 10 \mathrm{~cm}$, several abdominopelvic implants of over $2 \mathrm{~cm}$ and caecal wall infiltration.
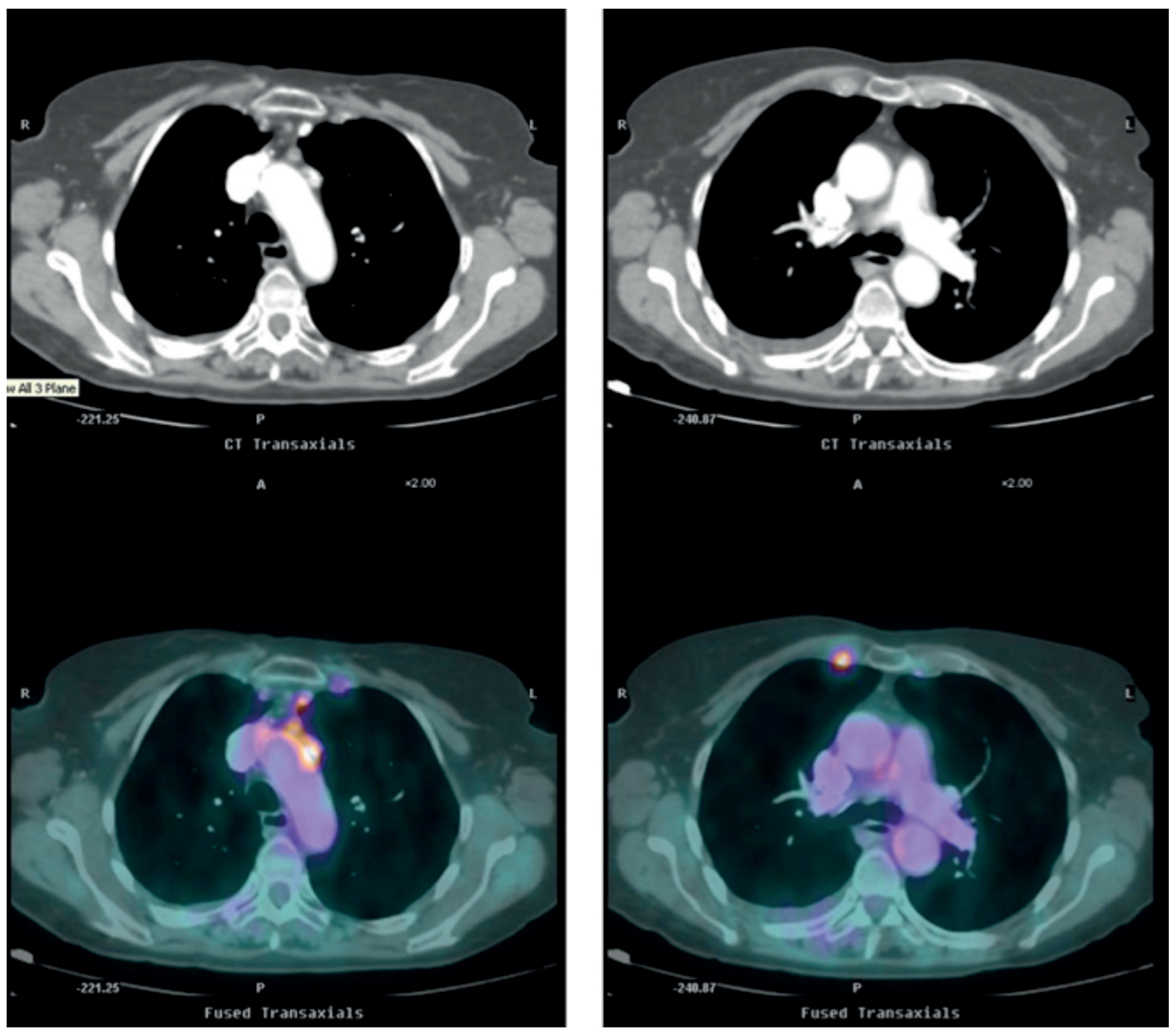

Fig. 2. Baseline positron emission tomography/computed tomography (PET/CT) scan: bilateral internal mammary lymph nodes as well as pre-vascular lymph nodes. 
forming a laparoscopy to confirm the diagnosis in order to evaluate if the patient could benefit from a primary debulking surgery (PDS).

The histopathological examination demonstrated lowgrade papillary serous ovarian cancer. Immunohistochemical study revealed tumor cells positive for CK7, WT1 and ER, marked heterogeneity of focally distributed p53-positive cells and a proliferation index Ki-67 between 15-20\% (Figure 3).

With the results obtained, the patient was presented at the MDT again, and it was agreed to perform a PDS.

The surgery included modified posterior exenteration with en bloc resection of the uterus, both adnexal masses, pelvic peritoneum, rectosigmoid colon, as well as peritonectomy of both parieto-colic spaces, radical omentectomy, splenectomy, pelvic and para-aortic lymphadenectomy, right diaphragmatic stripping and en bloc resection of a plaque of tumor.

The surgical pathology showed a low-grade papillary serous ovarian cancer affecting 27 out of 50 lymph nodes, extensive peritoneal carcinomatosis and infiltrating of all of the resected specimens.
Postoperative evolution was complicated by Serratia marcescens bacteremia and acute pancreatitis without severity criteria.

The CT scan carried out after the patient was discharged showed a small amount of perihepatic ascites, right pleural effusion and residual calcified cardiophrenic and bilateral internal mammary lymph nodes. CA-125 value was $829 \mathrm{IU} / \mathrm{mL}$.

We suggested adjuvant chemotherapy with carboplatin, paclitaxel and bevacizumab. After a thorough discussion about the treatment options, the patient finally declined chemotherapy. The patient was offered hormone therapy with letrozole, which she finally accepted.

A trimestral follow-up has been undertaken for the last 18 months, to date with no signs of progressive disease and stable tumor markers.

\section{Discussion}

Clinical management of women with low-grade ovarian cancer currently remains identical to that of other epithelial ovarian cancer histological subtypes. Undoubtedly, primary surgery is the backbone treatment of advanced

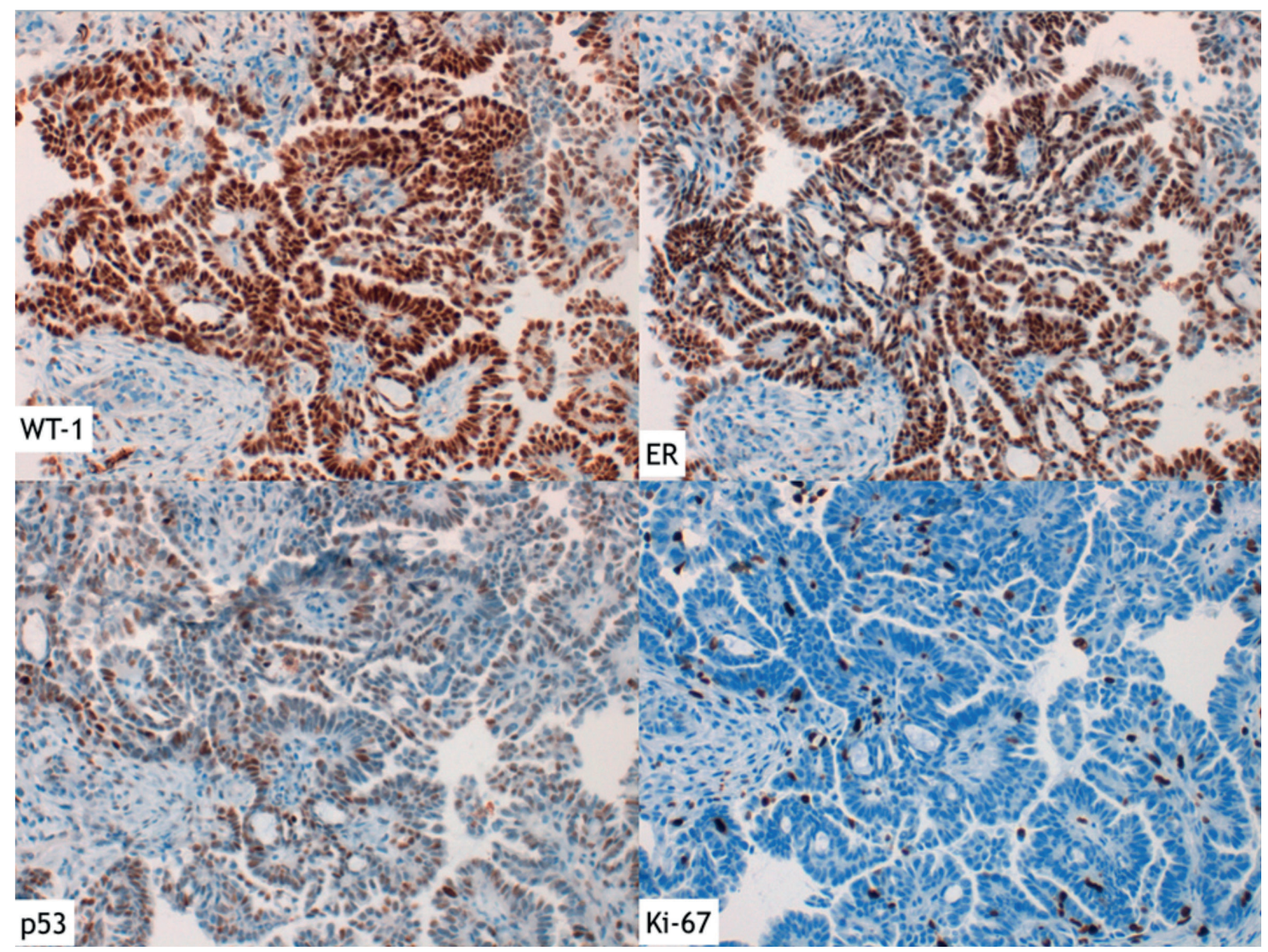

Fig. 3. Immunohistochemistry shows tumor cells positive for WT1 and estrogen receptors (ER), heterogeneous and focally distributed p53-positive cells and a proliferation index Ki-67 between 15-20\%. 
epithelial ovarian cancer after the initial assessment by a team experienced in gynecological oncology. However, we must be aware that some subtypes as LGSOC, clear cell or mucinous ovarian cancer behave distinctly differently from HGSOC, and this should be taken into consideration when planning the treatment.

Some evidence in the literature suggests that the role of surgery could be even more important in the initial management LGSOC. A retrospective trial reported that only $52 \%$ out of 112 stage II-IV LGSOC patients was clinically disease-free after completing the primary treatment, while in HGSOC this reached 70-80\% [4], probably due to lack of chemotherapy response. In addition, Crispens et al. described the association between optimal cytoreduction and overall survival (OS) [8].

Regarding systemic therapy, the standard of care consists of the administration of systemic chemotherapy with the combination of paclitaxel and carboplatin after the primary debulking surgery. In the specific context of LGSOC, two additional modalities have gained attention in the last years: the administration of bevacizumab and hormone therapy.

As the number of patients with a diagnosis of LGSOC included in the Gynecologic Cancer Intergroup (GCIG) International Collaboration on Ovarian Neoplasms 7 (ICON7) and the Gynecologic Oncology Group study 0218 (GOG-0218) was low, data for bevacizumab in first line treatment is limited $[9,10]$. In the context of recurrent disease, two retrospective studies from the MD Anderson Cancer Center and Memorial Sloan Kettering Cancer Center have demonstrated the activity of bevacizumab with or without chemotherapy in relapsed LGSOC or peritoneal cancer, with a clinical benefit in $77.5 \%$ and $73.3 \%$, respectively $[11,12]$. Among the two studies, bevacizumab monotherapy was administered to a total of 5 patients; 3 patients had stable disease, and 2 were not evaluable [11, 12].

Estrogen and progesterone are regulators of differentiation and growth of the ovaries that develop their biological activity through ER and progesterone receptors (PR). Compared with normal ovarian tissues, in ovarian cancer ER are upregulated, and PR expression is considered a good prognostic marker [13]. ER $\alpha$ is the isoform that, in ovarian cancer as in breast cancer, is most distinctly related to hormonal treatment sensitivity [13]. The Ovarian Tumor Analysis Consortium study included 2933 patients of different histological subtypes in order to analyze the prognostic role of hormonal receptors. However, with just 110 patients with LGSOC, no association between ER, PR and survival could be established [14]. Approximately $80 \%$ of LGSOC have high levels of ER and PR, suggesting these could respond to hormonal manipulation. Trials about the antiproliferative effects of hormonal therapy are currently lacking [15]. A recent retrospective trial examining the outcomes of hormone therapy with letrozole (received by $54.3 \%$ of patients), tamoxifen (28.6\%), anastrozole $(2.9 \%)$, leuprolide acetate $(7.1 \%)$ or leuprolide acetate with either letrozole, tamoxifen or medroxyprogesterone acetate $(7.2 \%)$ as maintenance treatment after platinum-based chemotherapy in stage II-IV LGSOC patients warranted a prospective trial, as the progression-free survival of those on treatment was 64.9 months compared with 26.4 months of those on observation $(p<0.001)$. Nevertheless there was no statistically significant benefit in OS (115.7 vs 102.7 months; $\mathrm{p}=0.42$ ) [16]. These results should be the seed of a new trial comparing hormonal therapy to platinum-based adjuvant chemotherapy.

For now, the mainstay of treatment for recurrent disease should remain surgical management, as the tumor is relatively chemoresistant [17]. In the recent years, some trials targeting the RAS-RAF-MEK pathway have obtained promising results. However, these preliminary data have not been confirmed in randomized clinical trials.

\section{Acknowledgments}

We thank the Pathology and Radiology departments of the MD Anderson Cancer Center, Madrid, for their continued support and use of the pathology and radiology images.

The Authors thank Ray Hill, an independent medical writer, who provided native English editing and journal styling on behalf of HPS. This editorial assistance was funded by PharmaMar, Spain.

\section{Conflicts of Interest}

The Authors declare there are no conflicts of interest in relation to this article. 


\section{References}

1. Plaxe SC. Epidemiology of low-grade serous ovarian cancer. Am J Obstet Gynecol 2008;198(4):459.e1-8.

2. Malpica A, Deavers MT, Lu K et al. Grading ovarian serous carcinoma using a two-tier system. Am J Surg Pathol 2004;28(4):496-504.

3. Kurman RJ, Carcangiu ML, Herrington CS, Young RH, editors. WHO Classification of Tumors of Female Reproductive Organs, 4th ed; 2014.

4. Gershenson DM, Sun CC, Lu KH et al. Clinical behavior of stage II-IV low-grade serous carcinoma of the ovary. Obstet Gynecol 2006;108(2):361-8.

5. Schmeler KM, Sun CC, Bodurka DC et al. Neoadjuvant chemotherapy for low-grade serous carcinoma of the ovary or peritoneum. Gynecol Oncol 2008;108(3):510-4.

6. Sieben NL, Macropoulos P, Roemen GM et al. In ovarian neoplasms, BRAF, but not KRAS, mutations are restricted to low-grade serous tumours. J Pathol 2004;202(3):336-40.

7. Silva EG, Gershenson DM, Malpica A et al. The recurrence and the overall survival rates of ovarian serous borderline neoplasms with noninvasive implants is time dependent. Am J Surg Pathol 2006;30(11):1367-71.

8. Crispens MA, Bodurka D, Deavers $M$ et al. Response and survival in patients with progressive or recurrent serous ovarian tumors of low malignant potential. Obstet Gynecol 2002;99(1):3-10.

9. Burger RA, Brady MF, Bookman MA et al. Incorporation of bevacizumab in the primary treatment of ovarian cancer. N Engl J Med 2011;365(26):2473-83.
10. Perren TJ, Swart AM, Pfisterer J et al. A phase 3 trial of bevacizumab in ovarian cancer. N Engl J Med 2011; 365(26):2484-96.

11. Dalton HJ, Fleming ND, Sun CC et al. Activity of bevacizumab-containing regimens in recurrent low-grade serous ovarian or peritoneal cancer: A single institution experience. Gynecol Oncol 2017;145(1):37-40.

12. Grisham RN, Iyer G, Sala E et al. Bevacizumab shows activity in patients with low-grade serous ovarian and primary peritoneal cancer. Int J Gynecol Cancer 2014;24(6):1010-4.

13. Lee $\mathrm{P}$, Rosen DG, Zhu C et al. Expression of progesterone receptor is a favorable prognostic marker in ovarian cancer. Gynecol Oncol 2005;96(3):671-7.

14. O’Donnell AJM, MacLeod KG, Burns DJ et al. Estrogen receptor mediates gene expression changes and growth response in ovarian cancer cells exposed to estrogen. Endo Rel Cancer 2005;12:851-66.

15. Sieh W, Köbel M, Longacre TA et al. Hormone-receptor expression and ovarian cancer survival: an Ovarian Tumor Tissue Analysis Consortium study. Lancet Oncol 2013; 14:853-62.

16. Gershenson DM, Bodurka DC, Coleman RL et al. Hormonal maintenance therapy for women with low-grade serous cancer of the ovary or peritoneum. J Clin Oncol 2017; 35(10):1103-11.

17. Gershenson DM, Sun CC, Bodurka D et al. Recurrent lowgrade serous ovarian carcinoma is relatively chemoresistant. Gynecol Oncol 2009;114(1):48-52. 


\section{Commentary}

The case report by Sánchez-Lorenzo and González-Martin nicely highlights the typical presentation and features as well as the controversies surrounding low-grade serous carcinoma of the ovary or peritoneum. As noted, common attributes of this rare subtype include younger age, prolonged overall survival, and relative chemoresistance compared to high-grade serous carcinoma [1-3]. There is no indication that the patient underwent genetic testing, even though she had a sister with breast cancer. And, this is not universally recommended. In fact, although approximately $18 \%$ of women with highgrade serous carcinoma have a germline BRCA mutation, only about $6 \%$ of women with low-grade serous carcinoma do [4].

Surgery continues to play a major role in the management of low-grade serous carcinoma. This patient underwent a laparoscopy to determine resectability. Given the fact that neither host characteristics nor imaging modalities are reliable in predicting the extent of disease, this strategy is increasingly being practiced in triaging women to primary cytoreductive surgery versus neoadjuvant chemotherapy, albeit in the face of an ongoing controversy regarding the benefit of the latter approach [5, 6]. This controversy may be even more animated for women with this subtype in view of its relative chemoresistance and the reported low response rate in the neoadjuvant setting [7]. The tumor burden in this patient appears to have been particularly high given the radicality of her procedure and the pathological findings. Low-grade serous carcinoma is typically diagnosed in the stage III category, and infiltrative tumor in several sites in also quite common.

Because of the relative chemoresistance of this subtype, over the past decade a search for more effective therapy has evolved. Thus far, the focus has been on the anti-angiogenic drug, bevacizumab; agents that target the MAP kinase pathway; and hormonal therapies. The initial recommendation for this patient included bevacizumab. While bevacizumab is approved in combination with chemotherapy in the first-line treatment of ovarian cancer in Europe based on a progression-free survival benefit [8,9], it is not yet FDA approved in the United States in this setting. Of course, these trials included women with all subtypes of epithelial ovarian cancer. The only reported analysis of bevacizumab specifically in lowgrade serous carcinoma in the first-line setting found no associated advantage. However, this analysis was undoubtedly underpowered [10]. Nevertheless, there is considerable information indicating activity of bevacizumab for low-grade serous carcinoma in the salvage setting [11, 12].

Targeted agents have also demonstrated activity in this subtype. Farley et al. reported a 15\% response rate of the MEK inhibitor, selumetinib, in the treatment of 52 women with recurrent low-grade serous carcinoma of the ovary or peritoneum [13]. Unfortunately, there was no correlation of response with mutational status (having a KRAS or BRAF mutation in a subset of patients). Three second-generation trials of different MEK inhibitors have been conducted - MILO (NCT01849874), NCT01936363, and GOG-281 (NCT02101788). The first two were closed prematurely because of futility and toxicity, respectively, and we are awaiting reporting of results. It is estimated that GOG-281 will complete accrual in late 2017. Additionally, a phase I trial of selumetinib plus the PARP inhibitor, olaparib, is due to be activated at The University of Texas MD Anderson Cancer Center in the near future. The BRAF inhibitor, vemurafenib, has also been reported to demonstrate response in a woman with a BRAF-mutated low-grade serous carcinoma [14].

Hormonal therapy is clearly active in low-grade serous carcinoma as well. As noted by Sánchez-Lorenzo and Gonzalez-Martin, a high proportion of low-grade serous carcinomas are rich in estrogen and progesterone receptors [15]. We initially reported our experience with 64 women who received 89 separate hormonal therapies for recurrent low-grade serous carcinoma [16]. The overall response rate was $9 \%$, the stable disease rate was $62 \%$, and the median time to progression was 7.4 months. In addition, a retrospective study of hormonal therapy in the maintenance setting following primary platinumbased chemotherapy found a significant advantage in terms of progression-free survival compared to chemotherapy followed by observation - median, 65 versus 26 months, respectively [17]. Consequent$l y$, a series of trials involving hormonal therapy for low-grade serous carcinoma in the neoadjuvant, maintenance, and salvage settings are currently in various stages of development.

Despite the fact that platinum-based chemotherapy is the standard for women with metastatic low- 
grade serous carcinoma, this patient refused this option, which was likely based on available information regarding this subtype's relative chemoresistance. She chose letrozole monotherapy and is continuing to do well 18 months later without disease progression. Increasingly, oncologists and patients are choosing to forego chemotherapy for the alternative of hormonal therapy. In the US, the National Comprehensive Cancer Network (NCCN) guidelines include hormonal therapy as an option for initial treatment of women with low-grade serous carcinoma of all stages based on expert opinion [18]. Clearly, we need a randomized clinical trial comparing chemotherapy to hormonal therapy in the firstline setting to determine if there should, in fact, be a new standard.

David M. Gershenson

Department of Gynecologic Oncology \& Reproductive Medicine The University of Texas MD Anderson Cancer Center, Houston, Texas, USA E-mail:dgershen@mdanderson.org

\section{References}

1. Gershenson DM, Sun CC, Lu KH et al. Clinical behavior of stage II-IV low-grade serous carcinoma of the ovary. Obstet Gynecol 2006;108(2):361-8.

2. Gershenson DM, Bodurka DC, Lu KH et al. Impact of age and primary disease site on outcome in women with lowgrade serous carcinoma of the ovary or peritoneum: Results of a large single-institution registry of a rare tumor. J Clin Oncol 2015;20;33(24):2675-82.

3. Grabowski JP, Harter P, Heitz F et al. Operability and chemotherapy responsiveness in advanced low-grade serous ovarian cancer. An analysis of the AGO Study Group metadatabase. Gynecol Oncol 2016;140(3):457-62.

4. Norquist BM, Harrell MI, Brady MF et al. Inherited mutations in women with ovarian carcinoma. JAMA Oncol 2016;2(4):482-90.

5. Petrillo M, Vizzielli G, Fanfani F et al. Definition of a dynamic laparoscopic model for the prediction of incomplete cytoreduction in advanced epithelial ovarian cancer: Proof of a concept. Gynecol Oncol 2015;139(1):5-9.

6. Rutten MJ, van Meurs HS, van de Vrie R et al. Laparoscopy to predict the result of primary cytoreductive surgery in patients with advanced ovarian cancer: A randomized controlled trial. J Clin Oncol 2017;35(6):613-21.

7. Schmeler KM, Sun CC, Bodurka DC et al. Neoadjuvant chemotherapy for low-grade serous carcinoma of the ovary or peritoneum. Gynecol Oncol 2008;108(3):510-4.

8. Perren TJ, Swart A-M, Pfisterer J et al. A phase 3 trial of bevacizumab in ovarian cancer. N Engl J Med 2011;365(26): 2484-96.

9. Burger RA, Brady MF, Bookman MA et al. Incorporation of bevacizumab in the primary treatment of ovarian cancer. N Engl J Med 2011;365(26):2473-83.
10. Oza AM, Cook AD, Pfisterer J et al. Chemotherapy with or without bevacizumab for women with newly diagnosed ovarian cancer (ICON7): overall survival results of a phase 3 randomised trial. Lancet Oncol 2015;16(8):1-9.

11. Grisham RN, Iyer G, Sala E et al. Bevacizumab shows activity in patients with low-grade serous ovarian and primary peritoneal cancer. Int J Gynecol Cancer 2014;24(6):1010-4.

12. Dalton HJ, Fleming ND, Sun CC et al. Activity of bevacizumab-containing regimens in recurrent low-grade serous ovarian or peritoneal cancer: A single institution experience. Gynecol Oncol 2017;145(1):37-40.

13. Farley J, Brady WE, Vathipadiekal V et al. Selumetinib in women with recurrent low-grade serous carcinoma of the ovary or peritoneum: an open-label, single-arm, phase 2 study. Lancet Oncol 2013;14(2):134-40.

14. Hyman DM, Puzanov I, Subbiah V et al. Vemurafenib in multiple nonmelanoma cancers with BRAFV600 mutations. N Engl J Med 2015;373(8):726-36.

15. Sieh W, Kobel M, Longacre TA et al. Hormone-receptor expression and ovarian cancer survival: An Ovarian Tumor Tissue Analysis consortium study. Lancet Oncol 2013;14(9): 853-62.

16. Gershenson DM, Sun CC, Iyer RB et al. Hormonal therapy for recurrent low-grade serous carcinoma of the ovary or peritoneum. Gynecol Oncol 2012;125(3):661-6.

17. Gershenson DM, Bodurka DC, Coleman RL et al. Hormonal maintenance therapy for women with low-grade serous cancer of the ovary or peritoneum. J Clin Oncol 2017;35(10): 1103-11.

18. National Comprehensive Cancer Network. NCCN Clinical Practice Guidelines in Oncology (NCCN Guidelines $\left.{ }^{\circledR}\right)$ : Ovarian Cancer Version 2.2017 - June 27, 2017 [online]. 